\begin{tabular}{|l|l|l|l|l|}
\hline Cuadernos I. Geográfica & $20-21$ & pp. 65-83 & Logroño & 1994-95 \\
\hline
\end{tabular}

\title{
APROXIMACIÓN METODOLÓGICA A LA EVALUACIÓN DEL ESTADO DE EROSIÓN HÍdRICA EN EL ÁMBITO DE LA MONTAÑ̃ MEDITERRÁNEA. APLICACIÓN A LA CABECERA DEL RÍO VÉLEZ, (SISTEMAS BÉTICOS)
}

\section{J. PERLES ROSELLó}

RESUMEN: El artículo recoge los fundamentos teóricos, principales pasos de elaboración y resultados cartográficos de una propuesta metodológica para la estimación cualitativa y cuantitativa del estado erosivo en un geosistema de montaña.

ABSTRACT: This article shows the theorical problems, elaboration steps and cartographic results of a qualitative and quantitative erosion state estimation methodology purpose.

RESUMEÉ: Le travail analyse les foundaments théoriques, les étapes principales dans l'élaboration et les résultats cartograpbiques obtenus dans une proposition mêtodologique sur une approche cualitative en quantitative de l'état érosive dans un geósystème de montagne.

Palabras clave: Erosión, Cartografía Montaña.

Key words: Erosion, Cartography, Mountain.

\section{Introducción}

La modificación y consiguiente aceleración del ritmo natural con el que se desarrollan los procesos de erosión hídrica produce una ruptura de equilibrio en el sistema de consecuencias directas e inmediatas sobre el hombre y su actividad. Los efectos de la pérdida de suelo se traducen en la mayoría de los casos en alteraciones en el modo de vida humano de la zona afectada, o se pone de manifiesto de forma más catastrófica a través de los procesos de avenida e inundación.

La fragilidad del sistema se hace más evidente en ámbitos como el mediterráneo en el que las particularidades climáticas (irregularidad, variabilidad y concentración en el tiempo de la precipitación) definen el "comportamiento 


\section{J. PERLES ROSELLÓ}

pluviométrico de un clima que tiene como normal las desviaciones de sus variables metereológicas" (LÓPEZ BERMÚDEZ, 1990)

Esta fragilidad se incrementa cuando evaluamos los procesos erosivos en geosistemas de montaña. La acentuación por la altitud de los episodios tormentosos, el elevado nivel de base de los cursos, y las altas pendientes, inciden junto a factores de naturaleza humana, como el cultivo o la frecuencia de incendios, en la configuración de un compendio de riesgos directos e indirectos de alta efectividad.

Si a este conjunto de fuerzas agresoras oponemos la escasa tolerancia a la denudación que presentan estos sistemas, consecuencia de la tradición de hábitat y aprovechamientos esquilmantes, definitivamente la montaña mediterránea se define como el geosistema frágil por antonomasia: por esta misma razón, estos espacios se constituyen como los principales receptores de las tareas correctoras de ordenación.

Siendo nuestro objetivo último exponer los pasos de preparación de una propuesta metodológica para la elaboración de una cartografia del estado de erosión en ámbitos de montaña, en las páginas siguiente conceptualizamos, en primer lugar, la noción de fragilidad erosiva, marco más amplio en el que la evaluación del estado erosivo se inscribe. A continuación definimos el concepto de estado de erosión, especificando las matizaciones que el estado de actividad de los signos de erosión introduce en este concepto. Esto, junto al carácter cuantitativo o cualitativo de la información, da lugar a distintos tipos de resultados cartográficos. Estos documentos, recogidos en el siguiente apartado, ofrecen una utilidad específica en el contexto general de la evaluación de la fragilidad erosiva, paso final en el que la estimación del estado erosivo adquiere pleno sentido.

Precisados estos conceptos, finalmente se recogen los pasos y criterios empleados para la elaboración tanto del documento básico (Mapa de signos de erosión), como de los que de él se derivan, mostrando los resultados cartográficos obtenidos.

\section{Concepto de fragilidad erosiva}

El concepto de fragilidad erosiva aplicada al geosistema de la montaña mediterránea transmite con expresividad y de una forma sintética varios hechos a la vez: por un lado un estado de cosas, resultante de un proceso en el tiempo, y por otro, nos da referencias de la dinámica de comportamiento del mismo en una situación futura. En este sentido, la fragilidad erosiva es un concepto complejo, múltiple, que sólo se explica a partir de la consideración de aspectos diversos, confluyentes y complementarios, de la dinámica erosiva.

Sin embargo, son frecuentes las publicaciones que, obviando este carácter complejo que la idea de fragilidad lleva implícito, reducen los análisis a aspectos 
parciales del proceso erosivo proponeniéndolos, no obstante, como indicativos del concepto general de fragilidad. Así, en publicaciones como el Mapa de Estados Erosivos (ICONA, 1990) concebidas en el ámbito de la ordenación de recursos, y que presumiblemente pretenden plasmar la idea de fragilidad como paso hacia el establecimiento de medidas correctoras, se sustituye este concepto por un aspecto parcial, la velocidad a la que la erosión se manifiesta; la no consideración simultánea de aspectos como el estado erosivo heredado o la tolerancia a ser erosionado de los diversos suelos, convierte a la cartografía en un documento escasamente representativo de las distintas situaciones y, por ello, de una eficacia cuestionable.

En una breve recopilación bibliográfica de publicaciones que contemplen esta necesidad de aunar diferentes aspectos hacia la idea de fragilidad erosiva, hay que citar en primer lugar el ensayo metodológico propuesto por el ICONA(1982).

Esta metodología recoge aspectos relativos al estado y velocidad del proceso, así como la vulnerabilidad a la erosión. Sin embargo, la metodología no llega a considerar la evaluación conjunta de los mismos, esto es, no existe una voluntad final de sumar los resultados positivos o negativos en cada uno de los aspectos analizados hacia el concepto de fragilidad.

A diferencia de ésta, la metodología propuesta por ZUIDAM y CANCELADO (1977) acumula en un único resultado los valores negativos y positivos para variables que hacen referencia al estado, susceptibilidad y tolerancia a la erosión, sin que sin embargo se produzca una diferenciación específica de los aspectos, ni se calculen resultados parciales para cada uno de ellos.

La metodología más completa, en nuestra opinión, es la propuesta por la F.A.O. (1984). En ella se definen expresamente aspectos diferenciados para el análisis de la erosión, y lo que es más importante, se fija como objetivo último de la evaluación la adición de los resultados parciales para cada uno de los aspectos, para así dar lugar al concepto de peligro erosivo.

Los aspectos definidos por F.A.O. para la estimación del peligro de erosión son: estado y velocidad de la erosión, riesgo inherente, y presión humana, estableciendo la igualdad:

peligro de erosión $=$ estado + velocidad + riesgo + p.humana

Con base definitiva en la estructuración de F.A.O., nuestra propuesta añade un concepto más a la estimación del peligro de erosión, como lo es la tolerancia del suelo. Además, hemos desestimado la diferenciación de la presión humana como un concepto aparte, por considerar que ésta se ejerce a través de actua- 
ciones (cultivo, incendios, talas, construcción) cuyos resultados afectan a la protección vegetal o a la erodibilidad, por lo que están considerados ya al introducir sus modificaciones en el riesgo inherente a la erosión.

De este modo, nuestra propuesta definitiva para la definición del concepto de fragilidad erosiva se articula del siguiente modo:

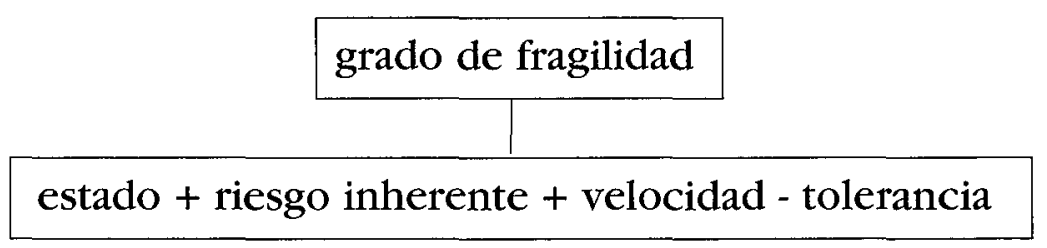

Se persigue como objetivo último la delimitación de zonas, a través de la acumulación de circunstancias adversas para cada uno de los aspectos parciales, en las que por concurrir un estado avanzado de erosión, un alto riesgo inherente a que la erosión se produzca, una constatación de una alta velocidad actual de la dinámica erosiva, y una escasa tolerancia del suelo a soportar esa erosión, la necesidad de protección en razón de la fragilidad del medio sea urgente.

Así, los conceptos esbozados en las líneas anteriores se definen finalmente como sigue:

Estado erosivo. Grado de intensidad de los signos de erosión manifestados en una zona y observados en una fecha concreta.

Velocidad de la erosión. Movilización de sedimentos por unidad de tiempo.

Riesgo inherente de erosión. Vulnerabilidad del terreno a ser erosionado en razón de las características físicas que lo conforman y condiciones naturales que le afectan.

Tolerancia a la erosión. Capacidad del suelo en razón de su estado, naturaleza y profundidad de soportar la erosión.

\section{Concepto de estado erosivo}

Definido el marco en el que consideramos adquiere pleno sentido la evaluación del estado erosivo, dos hechos matizan el significado de este concepto: el estado de actividad de los signos de erosión, y el carácter cualitativo o cuantitativo de la estimación. 
El primer factor de diferenciación viene dado por la noción de actividad en la dinámica erosiva. De este modo, habremos de distinguir los signos de erosión que presentan rasgos de actividad actual de aquellos cuya presencia en el terreno responde a crisis morfogenéticas anteriores, siendo su actividad erosiva nula o despreciable; esta matización conduce al concepto de estado erosivo activo frente al de estado erosivo inactivo. Una nueva matización nos lleva a individualizar los signos que aun respondiendo a una actividad morfogenética actual presentan una tendencia a la estabilización en razón de la práctica inexistencia de suelo o alteritas sobre los que se desarrollan, por lo que su evolución previsible avanza hacia el estacionamiento; este hecho se engloba en el concepto de signos de erosión con tendencia al estacionamiento.

El conjunto de todos estos signos de erosión, (signos activos, inactivos, y signos con tendencia a la estabilización), configura el concepto de estado de erosión, el cual da idea de la intensidad con la que estos signos, tanto de ablación como de sedimentación, han actuado sobre un territorio, manifestándonos el grado, leve o avanzado, de la degradación.

El matiz que la actividad o inactividad introduce en el concepto de estado erosivo diferencia tipos de información de diversa utilidad para la estimación de la fragilidad erosiva, concretamente para la estimación del riesgo de erosión, y de la tolerancia a la misma.

De este modo, para la deducción de cuáles son los factores de riesgo que se asocian a una mayor intensidad de los signos de erosión con objeto de establecer predicciones, habremos de partir, exclusivamente, de los signos de erosión que demuestren ser activos en la actualidad.

Además, el conocimiento de los signos de erosión con tendencia a la estabilización nos ayudará a comprender la evolución venidera de la actividad erosiva, puesto que pueden presentarse altos factores de riesgo erosivo sobre áreas con tendencia intrínseca a la anulación de la actividad.

Por otra parte, el conjunto de todos los signos, activos e inactivos, que definen el estado erosivo nos permitirá matizar el concepto de tolerancia, fundamentándolo no sólo en la profundidad y naturaleza del suelo, sino también en el estado de degradación que presente el terreno como herencia de la actividad de la erosión.

\section{Propuesta de cartografia derivada del concepto de estado de erosión. Mapas cualitativos, cuantitativos y de estado de actividad de la dinámica erosiva}

La diferenciación de matices hasta ahora expuesta en el concepto de estado erosivo se plasma cartográficamente en una serie de documentos (ver fig.1) de 
utilidad especifica para la obtención de otro aspectos conformantes de la fragilidad erosiva (tolerancia y riesgo). Esta serie de mapas completan nuestra propuesta para el análisis global del estado de erosión. En las siguientes lineas, adjuntamos una descripción de cada uno de los documentos, recogiendo el aspecto del estado erosivo que representan y la utilidad que poseen en el contexto de la fragilidad erosiva. Los pasos seguidos para la elaboración de cada documento se especificarán en el apartado siguiente.

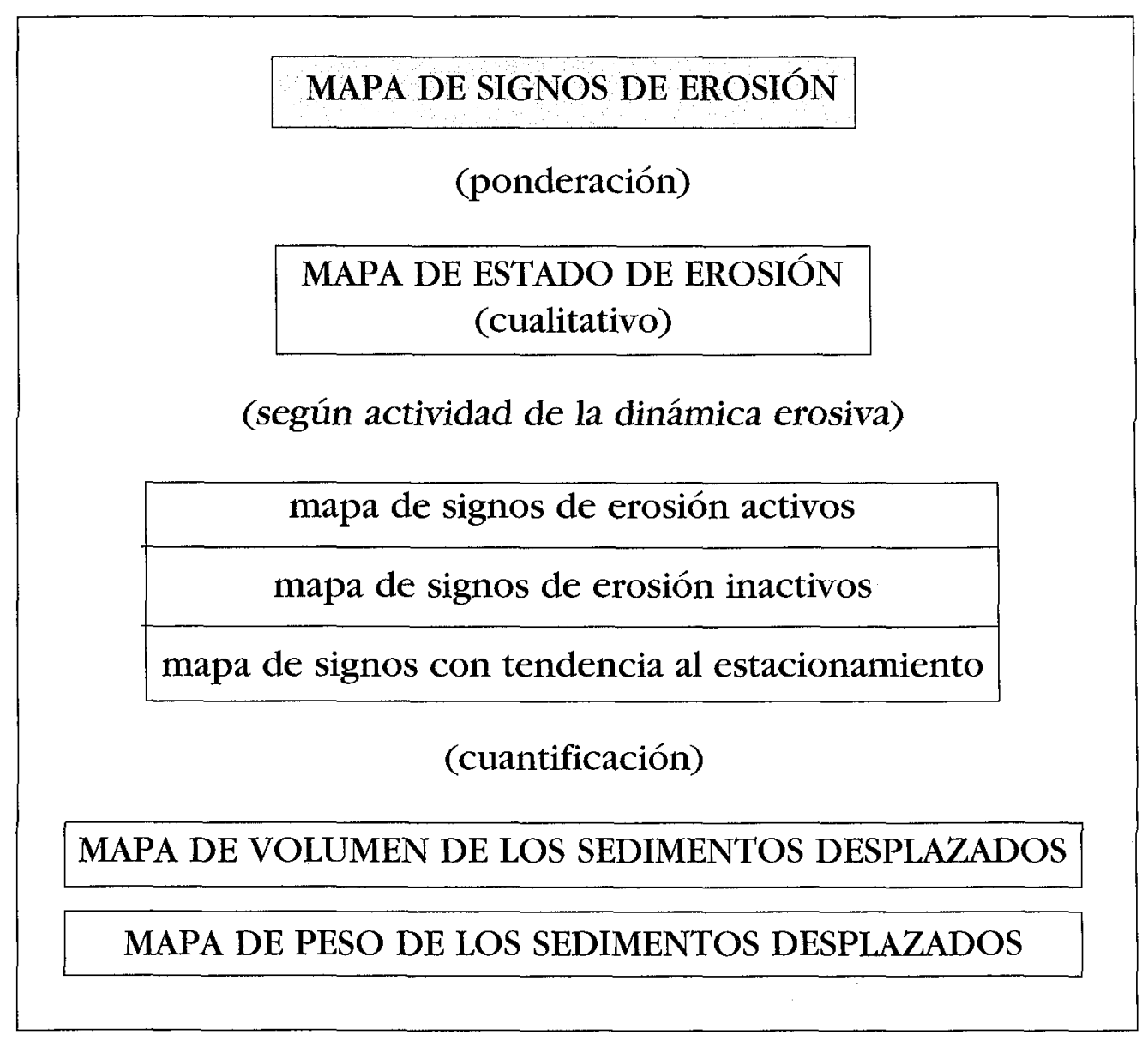

Figura 1: Cartografía deducida del Mapa de signos de erosión.

- Mapa de signos de erosión ( $n^{\circ}$ de cauces, erosión laminar y sedimentación). Constituye el documento primario y más general a partir del que se diferenciarán los restantes. Recoge la totalidad de los signos de erosión (activos, inactivos y con tendencia al estacionamiento), unificándolos en un solo valor de erosión para cada ladera mediante la adición de los distintos signos. El algoritmo utilizado para la unificación considera el hecho de que se trate de un signo de ablación o sedimentación. 
EVALUACIÓN DEL ESTADO DE EROSIÓN HÍDRICA EN EL ÁMBITO DE LA MONTAÑA MEDITERRÁNEA

\section{Documento resultante de la ponderación de los signos:}

- Mapa cualitativo de estado de erosión (signos de erosión ponderados). Recoge al igual que en el documento anterior la totalidad de los signos de erosión, ponderando la importancia de cada uno de ellos como agentes de degradación o sedimentación mediante un coeficiente proporcional a su magnitud, antes de unificarlos en un sólo valor de erosión. El documento (ver mapa 1), matiza el concepto de tolerancia.

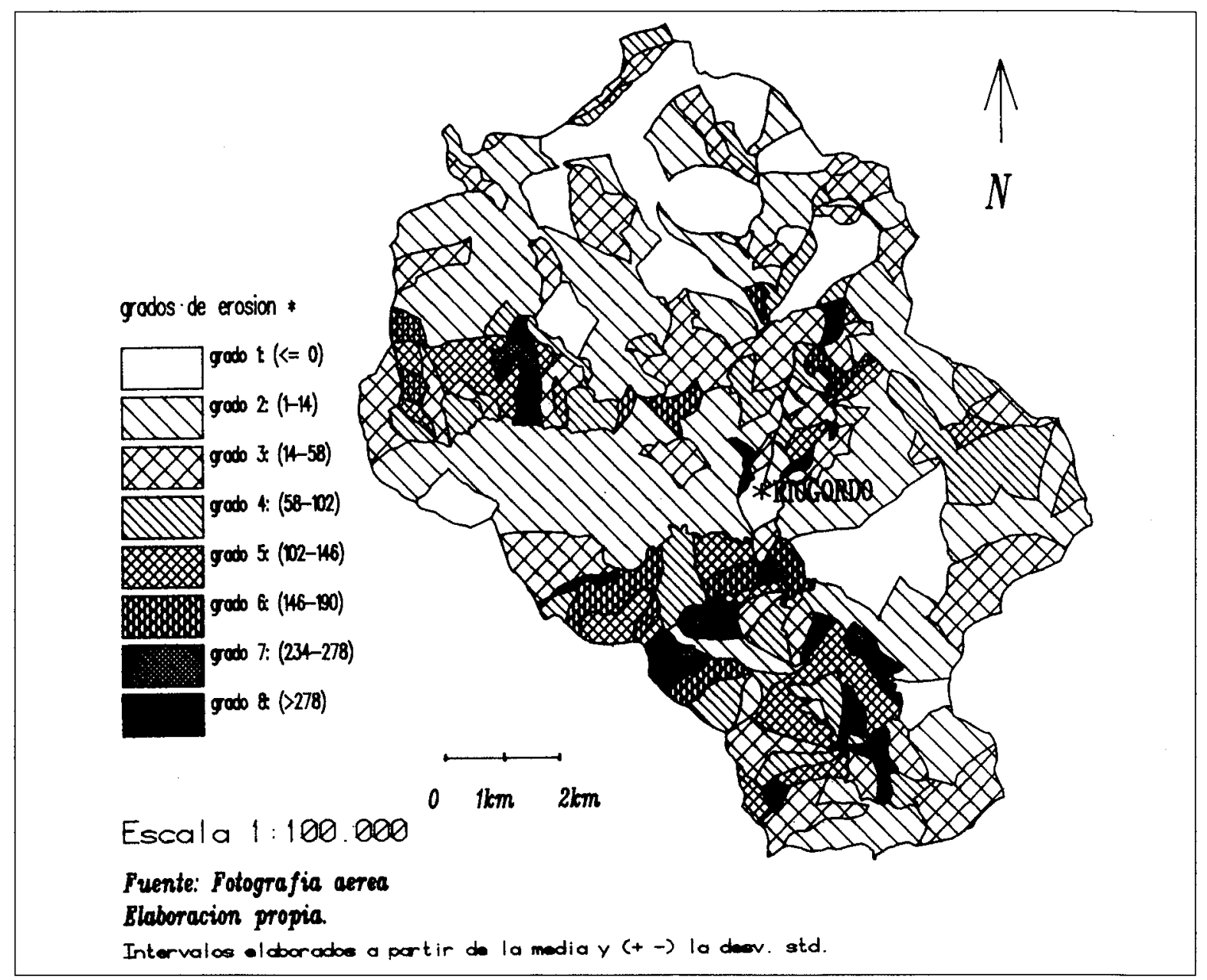

MAPA CUALITATIVO DE ESTADO DE EROSIÓN

Recoge signos de erosión activos, inactivos y con tendencia al estacionamiento. Da idea de la intensidad con que los signos de erosión (oblación y sedimentación) han actuado sobre el territorio. Matiza el concepto de tolerancia de erosión.

Las clases recogidas en la cartografía muestran una clara correspondencia entre las zonas de estado erosivo más avanzado (grado de erosión más elevado) con las litologías mas deleznables, las pendientes más acusadas y las áreas de menor porcentaje de cobertura de la vegetación

Mapa 1: Mapa cualitativo de estado de erosión (signos de erosión ponderados). 


\section{J. PERLES ROSELLÓ}

\section{Documentos diferenciados según el estado de la actividad erosiva:}

- Mapa de signos de erosión activos Recoge signos de erosión activos y con tendencia al estacionamiento. Constituye el fundamento para la delimitación de los factores responsables de la erosión (riesgo) y con ello para la predicción de la misma.

- Mapa de signos de erosión inactivos. Recoge signos de erosión originados en crisis morfoclimáticas anteriores y de nula actividad en la actualidad.

- Mapa de signos de erosión con tendencia al estacionamiento. Recoge signos de erosión instalados sobre suelos esqueléticos y con actividad limitada por condiciones de tolerancia. Son signos activos, pero con tendencia al estacionamiento de su dinámica por la escasez de suelo y alteritas sobre las que incidir. El documento contribuye a matizar la evolución futura de la actividad erosiva, en áreas en las que los factores de riesgo erosivo pueden ser altos, pero por contra la posibilidad de avance de la erosión escasa.

Documentos resultantes de la cuantificación del Mapa de signos de erosión:

- Mapa de volumen de sedimentos desplazado por unidad de superficie ( $\left.\boldsymbol{m}^{3} / \mathbf{h a}\right)$. (ver mapa 2 ) Es el resultado del cubicaje del volumen teórico de sedimentos desalojado para cada uno de los tipos de signos de erosión recogidos (erosión lineal, laminar y sedimentación).

- Mapa de peso de los sedimentos desplazados por unidad de superficie (Tm. /ha). Resultado de la multiplicación del volumen de sedimentos para cada unidad por la densidad media de litología sobre la que se desarrolla, obteniéndose para cada caso el peso de los sedimentos desalojados, cantidad que al igual que en el mapa anterior se ha relacionado con la extensión de cada ladera para hacer comparables los valores de las distintas unidades (ver mapa 3) 


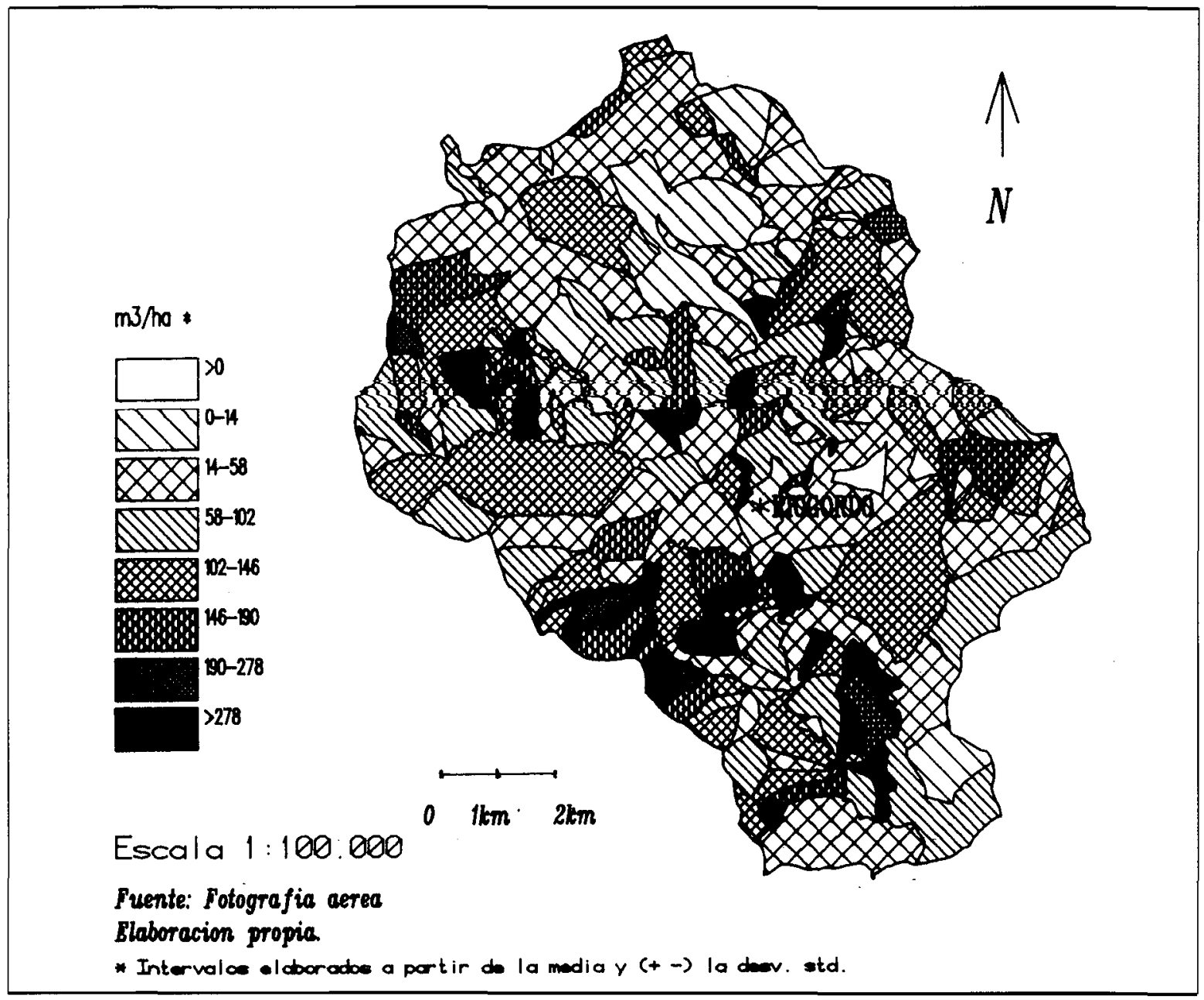

MAPA DE VOLUMEN DE TIERRA DESPLAZADA POR UNIDAD DE SUPERFICIE

Resultado del cubicaje del volumen teórico de sedimentos desalojado para cada una de las categorías de signos recogidas, utilizando para el cálculo valores prototípicos para la profundidad de los signos (ZUIDAM y CANCELADO, 1977), y reales, aunque medios, para la longitud de los cauces (longitud media de los cauces por cuenca, recogida en SENCIALES, 1992).

La cartografía refleja de nuevo la relación expresada en el mapa 1, acentuándose en esta ocasión el volumen de tierra desalojado para las cuencas con una longitud media de los cauces más elevada.

Mapa 2: Mapa de volumen de tierra desplazada por unidad de superficie $\left(\mathrm{m}^{3} / \mathrm{ha}\right)$. 


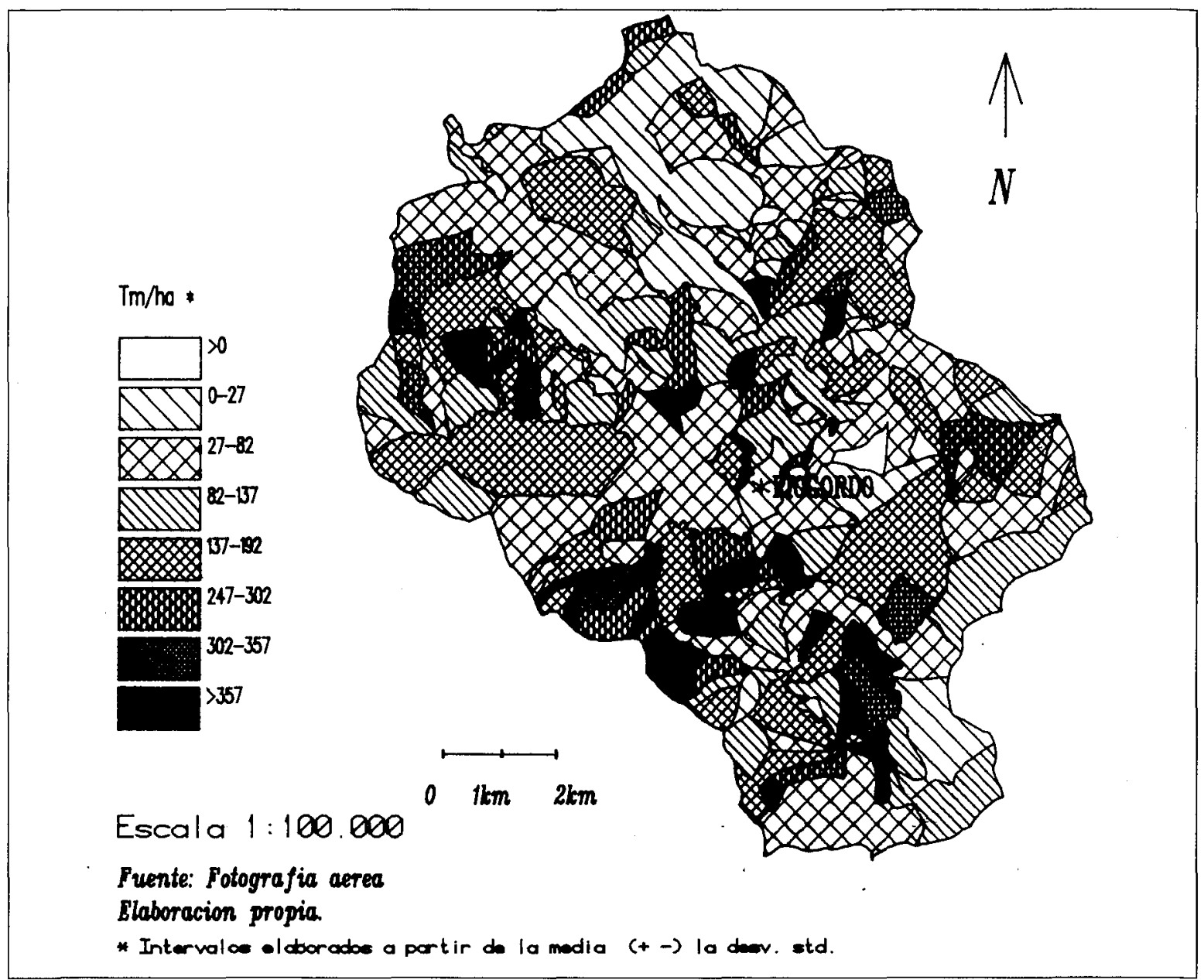

\section{MAPA DE PESO DE TIERRA DESPLAZADA POR UNIDAD DE SUPERFICIE}

Resultado de la multiplicación para cada unidad del volumen de sedimentos por la densidad media de la litología sobre la que se desarrolla, obteniéndose para cada caso el peso de los sedimentos desplazados por ladera y unidad de superficie.

El mapa muestra de nuevo una apreciable relación entre las zonas de estado erosivo avanzado con las variables de litología, pendiente, y cobertura de la vegetación, aumentando en este caso el tonelaje para las litologías de densidad media más alta.

Mapa 3: Mapa de peso de tierra desplazada por unidad de superficie (Tm./ha). 


\section{Metodología para la elaboración de la serie de mapas de estado erosivo en ámbitos de montaña}

\section{Recogida de información}

Para la elaboración de la serie de documentos cartográficos propuestos, hemos partido de la producción en primer lugar del Mapa de signos de erosión, base más general a partir de la cual individualizar los mapas derivados.

Nuestro objetivo ha sido el de representar el estado del terreno respecto a la intensidad con la que ha sido modificado por los agentes de erosión, manifestada a través de la cantidad de signos de erosión hídrica presentes en el territorio.

Elegimos una fuente (fotografía aérea $e=1: 30.000$ ) que nos permitiera utilizar una escala media de trabajo, que posibilitara un detalle suficiente para la observación, a la vez que la capacidad de circunscribir una extensión de terreno representativa $\left(87 \mathrm{~km}^{2}\right)$. De esta manera es factible afrontar la medición sin necesidad ni de muestrear ni de hacer extrapolaciones, pautas éstas de trabajo comunes a otras cartografías de erosión a nuestro entender altamente arriesgadas en un contexto tan variable como el de la montaña mediterránea. Junto a la información empírica que ha suministrado esta vía de observación/cuantificación, nos hemos apoyado también en una vía de información teórica que ha permitido formular hipótesis parciales de modelización del funcionamiento de la erosión en el micro-sistema de la ladera, complementando la información recogida mediante la observación.

En este contexto, basándonos en la fotografía aérea, recogimos datos cuantificados acerca del número de signos de erosión lineal enclavados en las laderas ( $\mathrm{n}^{\mathrm{o}}$ cauces colectores permanentes).

Basamos la elección de estas variables como indicadoras del estado de erosión en las recomendaciones expresadas por FAO (1984), que precisan: "La manera práctica más exacta de obtener estimaciones de la erosión hídrica consiste en combinar la fotografia aérea a baja cota con las estimaciones sobre el terreno . Mediante la interpretación de las fotografias aéreas se pueden hacer cálculos razonablemente satisfactorios del tamaño de los surcos y las cárcavas.".

Por otra parte, en lo que respecta a la estimación del grado de erosión laminar, magnitud de observación más dificultosa, se combinó la observación indirecta con la directa, como propone igualmente FAO: "La erosión laminar se puede calcular sobre el terreno observando las diferencias de coloración - en ciertos suelos - debidas a la pérdida de suelo superficial. A veces la remoción de tierra de alrededor de los árboles y las rocas se puede determinar por el reconocimiento de los niveles anteriores de la superficie del suelo que se aprecian en unos y otras". 
En definitiva, siguiendo estas pautas, hemos obtenido datos cuantificados acerca de las variables recogidas a continuación (la diferenciación de tres niveles de erosión lineal sigue los criterios y magnitudes aportados por ZUIDAM y CANCELAD0, 1977)

número de surcos por ladera
número de cárcavas por ladera
número de barrancos por ladera
grado de presencia de signos de erosión laminar
zonas de sedimentación coluvial.

Para su localización a través de la fotografía aérea, se procedió en primer lugar a un proceso de toma de referencia en el campo, identificando cada una de las clases teóricas con casos en la realidad, al objeto de establecer modelos de comparación.

Para la estimación de la erosión laminar, se consideró la presencia de la misma de acuerdo con la aparición sobre el terreno de pavimentos y pedestales de erosión en una magnitud apreciable, así como de raíces con signos de acumulación en la cara que enfrenta a la pendiente, y de descarnamiento en la opuesta.

El trabajo de campo fue precedido por un trabajo de fotointerpretación en el que pudieron determinarse unos criterios de proclividad a este tipo de erosión. Dedujimos de la observación que las condiciones de homogeneidad, carencia de obstáculos a lo largo del perfil y pendiente poco acentuada, hacían menos propicia la concentración de los cauces, posibilitando la uniformidad de la lámina de agua, y con ello de la erosión laminar (PERLES ROSELLO, 1992). Esta hipótesis de trabajo sirvió para organizar y estructurar la observación directa, penosa en una superficie tan extensa.

La unidad considerada idónea en un geosistema de montaña para la toma de referencia en el recuerdo de los signos de erosión fue la ladera. Entre las características ventajosas que presenta la ladera como unidad podemos citar:

- Constituye una unidad de homogeneidad suficiente al nivel de detalle escogido.

- Posee una unidad y dinámica de funcionamiento conjunto que le prestan un carácter de micro-sistema; este carácter permite modelizar su comportamiento en procesos como el de sedimentación (sedimentación coluvial, perfiles cóncavos). En este sentido, MINTEGUI (1991) señala como "existe una concatenación entre todos los elementos que la integran (..) que se 
EVALUACIÓN DEL ESTADO DE EROSIÓN HÍDRICA EN EL ÁMBITO DE LA MONTAÑA MEDITERRÁNEA

manifiesta en un equilibrio dinámico en donde una variación o alteración provocada en uno de ellos tiene efectos concatenados sobre el resto". En el mismo sentido IMESON (1984) afirma como "los ajustes hidrológicos en un lugar no pueden examinarse correctamente sin una referencia a los efectos de ajuste que ocurren ladera arriba y abajo".

Los criterios seguidos para la definición sobre el mapa de las laderas se fundamentan en los utilizados por ICONA (1988).

Procedimos a la delimitación de las laderas en una escala (1:10.000) más detallada que la propuesta en el método, para subsanar el problema del encubrimiento de subladeras. Se utilizaron para ello los mapas topográficos editados por la Junta de Andalucía, y se aumentó el apoyo prestado por la fotointerpretación para la comprobación de la delimitación. Se llegó así a un nivel de aproximación en el que el balance entre la perdida de precisión que el propio método conlleva (encubrimiento de pequeñas rupturas de pendiente) y la operatividad que el mismo oferta, se saldó como positivo a favor de esta última.

Una vez definidas las laderas se precedió a su digitalización y procesamiento a través de un Sistema de Información Geográfica, instrumento que nos ofrecía directamente el área estimada sobre el mapa para cada unidad, con lo que se posibilitaba la referenciación de las distintas magnitudes recogidas a una unidad de superficie $\left(\mathrm{m}^{3} / \mathrm{ha} \mathrm{Tm} . / \mathrm{ha}\right.$ etc.).

Dado que la superficie representada en el documento cartográfico, y por tanto transferida a través de la digitalización, es el resultado de la proyección ortogonal de la superficie real de cada una de las laderas, corregimos los valores de superficie cartográfica de las distintas laderas mediante la operación:

\section{Area real $=$ area proyectada: cos de la pendiente de la ladera}

Obteniendo así el área real de las laderas, y con ello valores ajustados para la referenciación a unidades de superficie.

La información recogida de esta forma por observación, se ha complementado con correcciones provenientes de supuestos teóricos. Así, por ejemplo, se ha tenido en cuenta la variable forma de la ladera, ya que en virtud de los cambios de pendiente y más allá de ello, de la relación topológica establecida entre estos cambios, se condicionan dentro de la misma los procesos de denudación o sedimentación, considerando de este modo la ladera como un pequeño sistema en el que tienen principio y fin muchos de los procesos erosivos. Modelos simples como el de KING (1962) se fundamentan en esta idea, seccionando la ladera en regiones fisiográficas asociadas a un determinado proceso hídrico. 


\section{J. PERLES ROSELLÓ}

En esta línea, habiendo analizado una relación entre los perfiles cóncavos, cóncavo-convexos y rectilineos de las laderas, con los procesos de sedimentación en último tramo del perfil (PERLES ROSELLO, 1992), utilizamos esta proposición para la localización de pequeñas áreas al pie de las laderas que presentaban los perfiles citados y pendiente situada por debajo de la pendiente de arrastre, entendiendo que por su situación topológica en el conjunto de la ladera se trata de áreas de depósito coluvial, en las que los procesos de sedimentación predominan sobre los de ablación, con la correspondiente alteración del significado de la gravedad del estado erosivo (mejores posibilidades para la pedogénesis, mejora de los niveles de tolerancia).

Recogidos de este modo la presencia o ausencia de signos de erosión presentes en cada ladera, y, en su caso, contabilizados los mismos, procedimos a la unificación de estos datos para la obtención de un único valor indicativo de erosión.

Criterios para la adición de los signos de erosión en razón de su carácter de ablacion o sedimentación.

Habiéndose recopilado información acerca de signos de erosión de diversa índole (lineal, laminar) y diferente significado (ablación /sedimentación) para la estimación del estado de degradación de la zona, se procedió a su unificación.

Dado que la metodología para la elaboración de la cartografia se fundamenta en la acumulación de puntuaciones para los diversos signos de erosión hacia un valor final que represente la gravedad del proceso en cada ladera, mediante el uso del algoritmo pertinente para la unificación, dotaremos a la información hasta ahora recopilada de un significado conjunto.

La principal diferenciación viene dada por el carácter agresor o protector de la variable recogida, aspecto que sitúa en un plano las variables número de surcos/cárcavas /barrancos por ladera y grado de erosión laminar, frente a la variable zonas de sedimentación. De este modo, habrá que introducir los valores de esta última con un signo negativo en el algoritmo, por su capacidad de contrarrestar la gravedad de la degradación. El algoritmo queda recogido en el cuadro 1.

Pautas para la elaboración de los mapas derivados. Ponderación, determinación de la actividad de los signos y cuantificación.

\section{a. Ponderación y cualificación}

El Mapa cualitativo de estado de erosión se fundamenta en la necesidad, de unir a los valores de frecuencia para cada signos, la diferente capacidad erosiva, en razón de su magnitud, de cada uno de estos signos. Así, los signos fue- 
EVALUACIÓN DEL ESTADO DE EROSIÓN HÍDRICA EN EL ÁMBITO DE LA MONTAÑA MEDITERRÁNEA

ron ponderados mediante un coeficiente ajustado a la entidad de cada uno de ellos.

Para la elaboración de la ponderación, analizamos la relación de proporción existente entre el volumen de un surco, una cárcava y un barranco tipo, al que le suponíamos una longitud igual a 1 (por lo que el volumen se equipara al área de la sección de cada cauce); así podíamos comparar la proporción entre los volúmenes sin que ésta se viera afectada por la variación en la longitud. Para las medidas de profundidad y anchura se ha tomado el valor de la marca de clase de los intervalos de dimensiones reseñados por ZUIDAM y CANCELADO (1977). Así, basándonos en las medidas recogidas en la fig. 2, la proporción se obtuvo como especifica el siguiente ejemplo:

coef. aplicado = vol. de una cárcava de longitud 1: vol. de un surco de longitud 1 al $n^{o}$ de cárcavas (área de la sección del cauce)

(área de la sección del cauce)

Los valores así obtenidos se utilizaron definitivamente como coeficientes (tabla 1), ponderándose la importancia de las formas de erosión lineal de mayor entidad.

La necesidad de ponderación de los diversos signos se recoge también en la metodología de ICONA (1982), aunque, a nuestro entender, la arbitrariedad del criterio para la designación de los coeficientes, que secuencia la importancia de los signos pero sin considerar la proporcionalidad, la hace poco realista.

Basándonos definitivamente en la ponderación recogida en el cuadro $n^{\circ} 1$, y en el sentido del signo de erosión recogido (ablación o sedimentación), el algoritmo definitivo utilizado para la agrupación de todos los valores en un valor final de estado de erosión para cada ladera se recoge en la tabla 1:

Tabla 1: Coeficientes utilizados para la ponderación de los signos de erosión.

\begin{tabular}{|c|c|}
\hline \multicolumn{2}{|l|}{ grado estado erosivo } \\
\hline$+\mathrm{n}^{\circ}$ de surcos/ladera & ${ }^{*}$ coef. $(12,5)$ \\
\hline$+\mathrm{n}^{\circ}$ de cárcavas/ladera & "coef. $(75)$ \\
\hline$+\mathrm{n}^{\circ}$ de barrancos/ladera & ${ }^{*}$ coef. $(150)$ \\
\hline + erosión laminar & "coef. (área* $1 \mathrm{~cm}$.) \\
\hline - sedimentación & "coef. (área* $1 \mathrm{~cm}$.) \\
\hline
\end{tabular}


M. J. PERLES ROSELLÓ

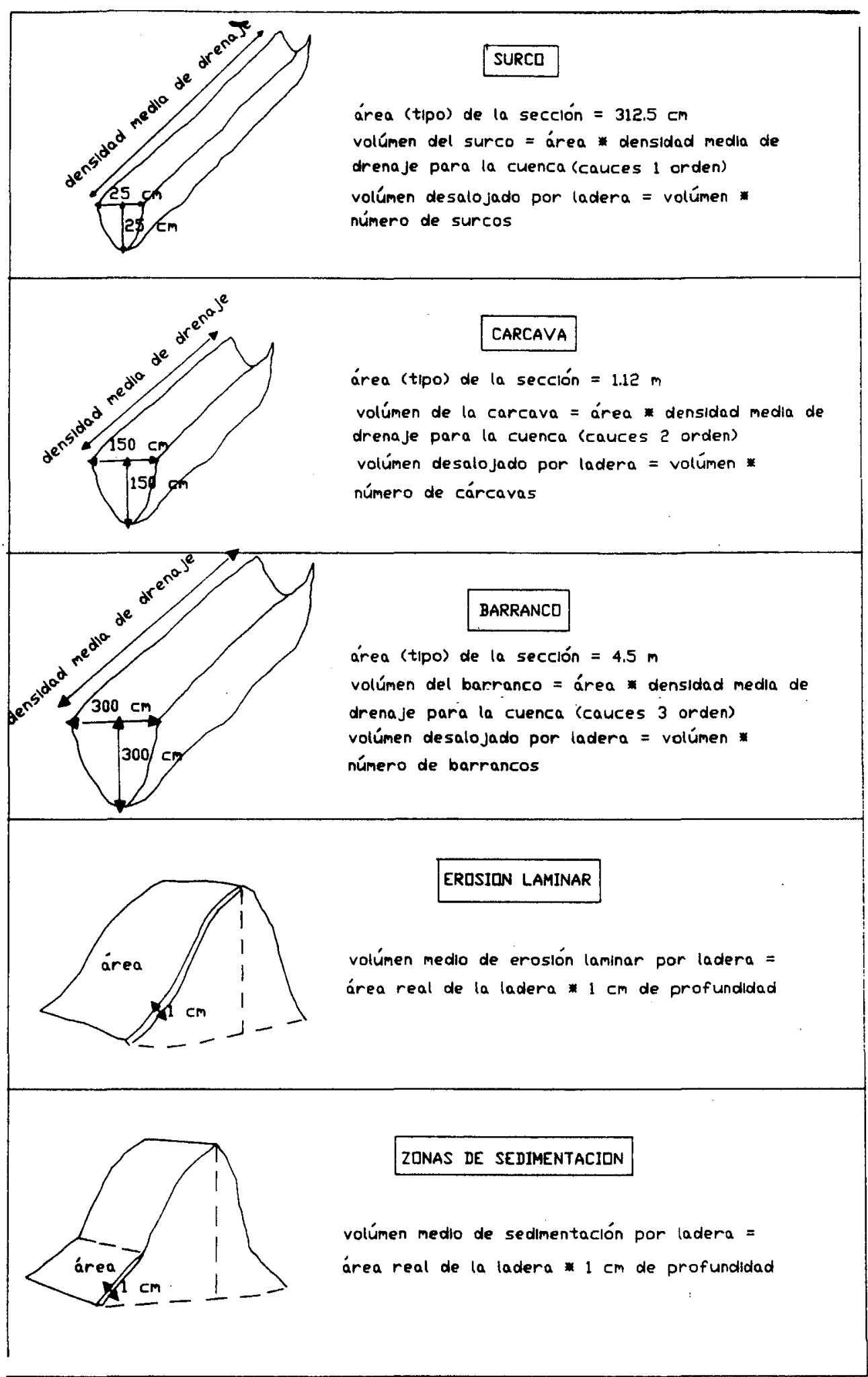

Figura 2: Medidas standar utilizadas para obtener los coeficientes de ponderación y para cubicar el volumen de tierra desalojada por cada signo de erosión. 
Mediante este algoritmo se obtuvo definitivamente el parámetro indicador del grado de erosión representado en el Mapa cualitativo de estado de erosión según los signos de erosión ponderados por unidad de superficie (ver mapa 1).

\section{b. Determinación del estado de actividad de los signos}

Partiendo nuevamente del Mapa de signos de erosión procedimos a la evaluación del grado de actividad erosiva de los signos recogidos, para generar de este modo el mapa de signos de erosión activo, inactivo y con tendencia al estacionamiento.

El Mapa de signos de erosión activos constituye el documento base para la predicción del riesgo erosivo, atendiendo al hecho de que una alta frecuencia de signos de erosión solo sería indicativa del riesgo de erosión si se comprobaba su actividad. IMESON (1984) recoge la idea al precisar: "En algunos casos los fenómenos de erosión son suficientes para proporcionar indicaciones de tendencias a la estabilidad y una advertencia del riesgo potencial de erosión, pero esas interpretaciones pueden ser falsas sin una apreciación de las condiciones geomorfológicas (..) En las regiones semi-áridas, las marcas de la erosión persisten durante centenares de años, creando un relieve que a primera vista parece estar sufriendo una erosión rápida y grave, pero que de hecho puede estar relativamente inactiva."

Para la tarea de la comprobación de la actividad actual de los signos de erosión se adoptaron como marco los criterios recomendados por la FAO (1984) a tal efecto, y mediante trabajo de campo se observaron los fenómenos siguientes:

- Discurrir de aguas fangosas y hojarasca superficial por las laderas o cauces.

- Presencia de signos de erosión que por su escasa perdurabilidad en el tiempo lleven implícito el carácter reciente de su formación (pavimentos y pedestales de erosión, adherencias de tierra a vegetación estacional)

- Comprobación de la actividad de los barrancos, considerando que la actividad de estos colectores de mayor entidad lleva implícita la de sus tributarios surcos y cárcavas; así, se observaron las características del lecho de estos cauces (presencia de plantas no estacionales, acumulaciones de sedimentación de granulometría fina, alteración del perfil del lecho por depósitos provenientes de las márgenes y no evacuados).

- Comprobación de la leve dinámica erosiva (escasez de depósitos, cauces cuasi estacionarios) de los signos enclavados sobre litosuelos o suelos esqueléticos. 


\section{J. PERLES ROSELLÓ}

\section{c. Cuantificación}

En lo que respecta al procedimiento de transformación de los datos primarios de frecuencia de los signos de erosión en valores de volumen y peso (Mapas 2 y 3), el paso esencial lo constituyó el cubicaje, utilizando mediciones tipo, del volumen teórico de tierra desalojada o en su contra, depositada, por los diversos signos de erosión (procedimiento similar al utilizado por FERRE y SENCIALES (1990). En este caso se ha utilizado para el cálculo valores prototípicos (ver fig. 2) para el ancho y la profundidad de los signos (ZUIDAM y CANCELAD0,1977) y reales aunque medios, para la longitud de los cauces (densidad media de drenaje por cuencas, recogida en este caso de SENCIALES,1992). Para el cálculo del volumen desalojado por la erosión laminar o depositado por la sedimentación se ha estimado como valor representativo el área de la zona afectada por $1 \mathrm{~cm}$. de profundidad.

Calculado en razón de estas medidas el volumen de tierra desalojado por cada signo, se multiplicó el mismo por el número de signos presentes en cada ladera ( $\mathrm{m}^{3}$ desplazado por ladera). Al objeto de hacer los valores comparables, los resultados se relacionaron con el área real de cada ladera, resultando un valor final de $\mathrm{m}^{3}$ /ha para cada ladera (Ver mapa 2).

Los valores de volumen obtenidos por ladera, fueron estimados en peso Ver mapa 3) mediante la multiplicación del valor de volumen por el de la densidad de la litología predominante en la misma, y nuevamente relacionados con el área (Tm./ha).

\section{Resultados cartográficos y conclusiones}

El documento cartográfico básico (mapa 1) refleja con gran efectividad un ajuste entre los grados de erosión y las principales unidades litológicas y de paisaje presentes en la zona, mostrándose los siguientes hechos:

- Correspondencia de las zonas de alto grado de erosión con la variable litológica. Es posible diferenciar los valores medios de estado erosivo arrojados para cada litología, siendo las medias más elevadas las correspondientes a los materiales pizarrosos altamente tectonizados de la Unidad de los Montes de Málaga, (sector suroriental del mapa) y a la franja central de brechas del mismo origen (franja este-oeste al norte de Riogordo).

- Relación de las zonas de alto grado de erosión con otras variables indicadoras de riesgo de erosión como son las altas pendientes (mogotes calizos de la Sierra del Rey) y el bajo nivel de cobertura vegetal. Por contra las zonas de pendientes suaves (litología margosa- areniscosa) y pendientes suaves al sur de la franja de brechas (fondo del pasillo de Colmenar) y al norte de la misma (piedemonte de la Sierra de las Cabras), poseen los valores de estado erosivo más moderados. 
A la luz del elevado grado de correspondencia observado entre la zonificación obtenida en el mapa y las principales unidades de paisaje, concluimos la efectividad del procedimiento metodológico llevado a cabo para su elaboración. Se estiman igualmente como positivos el nivel de precisión obtenido en la plasmación cartográfica del fenómeno, así como la posibilidad de comparación de resultados, tanto de las clases cualitativas como cuantitativas.

\section{Referencias bibliográficas}

FAO-PNUMA (1984): Metodología provisional para la evaluación y representación cartográfica de la desertización. Roma.

FERRE,E. y SENCIALES, J.M. (1990): Notas sobre la erosión hídrica en las laderas de los montes de Málaga. Reunión Nacional de Geomorfología. Sociedad Española de Geomorfología. Teruel.

ICONA (1982): Paisajes erosivos en el sureste español. Ensayo de metodología para el estudio de su cualificación y cuantificación. MAPA, Proyecto LUCDEME. Madrid.

ICONA (1988): Atlas de laderas y pendientes de las cordilleras Béticas Litorales.

ICONA (1990): Mapas de estados erosivos. Cuenca bidrográfica del Sur, MAPA. Madrid.

IMESON A.C. (1984): Una vía de ataque eco morfológica al problema $b$ de la degradación y erosión del suelo. Desertificación en Europa. Monografías de la D.G.M.A, Madrid.

LÓPEZ BERMÚDEZ, F. y ALBADALEJO, J. (1990): Factores ambientales de la degradación del suelo en el área mediterránea. Degradación y regeneración del suelo en condiciones ambientales mediterráneas. CSIC. Murcia

KING ( 1962 ): The morphology of the earth. A study and sinthesis of world scenary. Ed Oliver \& Boyd. Edinburg, en CEOTMA (1982): Guía para la elaboración de estudios del medio físico, MOPU, Madrid.

MINTEGUI AGUIRRE, J.A. (1991): La ordenación agrohidrológica. Seminario sobre erosión y desertificación en Andalucía, Málaga (inédito )

PERLES ROSELLO, M.J. (1992): Relaciones forma de la ladera-patrón de drenaje y litología. Estudios de Geomorfología en España. Sociedad Española de Geomorfología. Murcia.

SENCIALES GONZALEZ, J.M. (1992): Análisis morfométrico de la Cuenca del río Benamargosa. Estudios de Geomorfología en España. Sociedad española de Geomorfología, Murcia .

VAN ZUIDAM y CANCELADO (1977): Terrain analysis and classification using aerial photographie. A geomorpbological aproach. ITC textbook of pboto interpretation. Vol VII. Netherlands. 
